\title{
Reproductive Dysfunction in Young Men with Epilepsy
}

\author{
Anna Voitiuk* and T Litovchenko \\ ${ }^{1}$ Department of Neurology and Child Neurology, Ukraine \\ ${ }_{2}^{2}$ Medical Centre "Neiron", Kharkiv, Ukraine
}

*Corresponding author: Anna Voitiuk, Department of Neurology and Child Neurology, Kharkiv Medical Academy of Postgraduate Education, Kharkiv, Ukraine.

Received Date: January 23, 2021

Published Date: February 09, 2021

\begin{abstract}
There are many studies and works devoted to the problems of epilepsy in women in neurology. However, this pathology is also observed in men, especially since the incidence in young men is slightly higher. Therefore, the purpose of this study was to assess the quality of life of young men with epilepsy, as well as to assess the effect of epilepsy and antiepileptic drugs on the reproductive system in men. Because this problem remains relevant today.
\end{abstract}

Keywords: Epilepsy; Young men; Quality of life; Reproductive dysfunction; Antiepileptic drugs

\section{Introduction}

Epilepsy is one of the most important clinical problems and ranks third among all organic brain diseases. According to the World Health Organization (WHO), more than 65 million people worldwide suffer from epilepsy. This pathology is diagnosed in five million people every year [1]. The 55 new cases per 100,000 people are reported annually in high-income countries. This rate can be more than twice as high (149 cases per 100,000 population) in lowincome and middle-income countries. The prevalence of epilepsy in Ukraine ranges from 50 to 73 cases per 100,000 population (an average of 73.9 per 100,000$)$. In addition, the average incidence in men (53.7 per 100,000) is slightly higher than in women (46.3 per 100,000) [2]. Epilepsy is a broad interdisciplinary problem that, in addition to neurology, is related to neurosurgery, psychiatry, endocrinology, sexology, and urology. The pathology of the reproductive system in men with epilepsy can manifest itself both in the form of decreased libido, decreased fertility, erectile dysfunction, orgasm and ejaculation disorders, and lead to infertility. A decrease in free testosterone levels contributes to this dysfunction [3-5].

Sexual activity itself can trigger an attack due to hyperventilation and activation of the sensory cortical region of the genitals. Sexual phenomena can also be part of an epileptic seizure, such as motor symptoms, such as erection, ejaculation, orgasm, or pelvic genital movements. The development of sexual disorders often leads to a violation of the function of limbic structures. Epileptiform discharges in the interventional period can interrupt the temporal-limbic modulation of the hypothalamic-pituitary function. Disorders of the epileptiform nature can stimulate or depress the hypothalamus. This leads to an increase or decrease in gonadotropin-releasing hormone. Such hormonal disorders lead to hyposexuality, decreased potency, hypogonadotropic hypogonadism, impaired sexual desire and sexual activation [6-11]. 
Epilepsy itself is an endocrine disruptor and leads to hypothalamus dysfunction, which, in turn, usually has the opposite effect on the concentration of androgens in young men, i.e., androgen activity decreases. The effect of epilepsy on central reproductive activity is complicated by the metabolic changes caused by antiepileptic drugs on reproductive hormones. Also, these diseases can coexist independently of each other, i.e. be comorbid, making the course of each pathology worse and worsening the quality of life and prognosis for the patient as a whole. The effect of antiepileptic drugs (AEDs) on the functioning of the reproductive system in men should be considered separately.

The enzyme-inducing drugs (phenobarbital, benzonal, phenytoin, carbamazepine) increase the metabolism in the liver, stimulating the cytochrome P450 system, and also accelerate the metabolism of steroid sex hormones. Accordingly, the level of testosterone decreases. But the main mechanism is due to the fact that enzyme-inducing drugs increase the concentration of globulins that bind sex steroids. Normally, about $40-45 \%$ of them bind to globulins, $53-55 \%$ - to albumins, and $2-3 \%$ are in the free state. Biologically active testosterone includes the free fraction and albumin-bound testosterone. When prescribing enzyme-inducing drugs, the concentration of binding globulins increases. As a result, the amount of inactive testosterone fraction increases and the free and albumin-bound (biologically active) fraction decreases. Thus, there is a relative decrease in testosterone levels [6].

Valproic acid is not an inducer of liver enzymes. Against the background of valproate therapy, a low level of follicle-stimulating hormone was determined, while the serum level of testosterone was normal [5]. The level of basal and induced serum prolactin in males who took valproate was also within the normal range $[4,7,9]$. However, there are cases of infertility among young men who took valproate in the treatment of epilepsy [12]. Levetiracetam, which binds to the SV2A receptor present in the brain, gonadal tissues, and the endocrine system, was associated with an increase in total testosterone levels in young men one month after the start of treatment [13]. AEDs cause a decrease in serum testosterone levels and an increase in estradiol levels, which leads to sexual dysfunction in men with epilepsy.

AEDs modulate the release of hormones from the hypothalamuspituitary-gonadal axis (Figure 1) and can have a direct inhibitory effect on sexual and reproductive function (Figure 1).

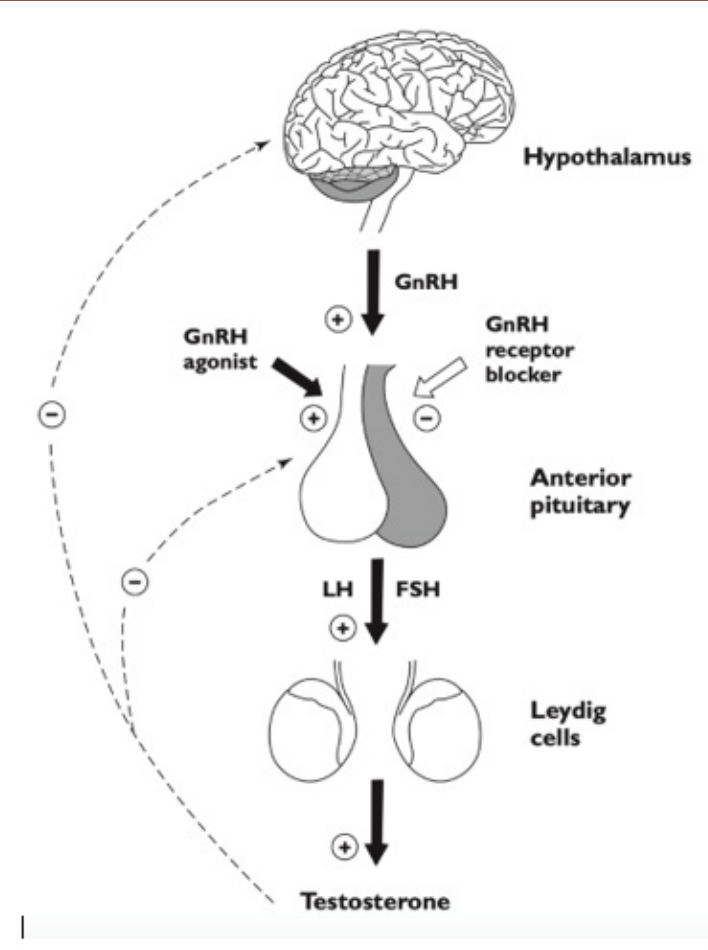

Figure 1: Schematic representation of the male hypothalamic-pituitary-gonadal (HPG) axis.

They can alter the metabolism of steroid sex hormones and their binding proteins. The decreased fertility and impairment of various aspects of sexual function are common in young men with epilepsy. The resulting neuroendocrine effects with changes in steroid sex hormones can alter epileptogenicity [10]. The purpose of the study is to evaluate the effect of epilepsy and antiepileptic drugs on male reproductive function, as well as to investigate the features of the influence of this pathology on the quality of life of patients with epilepsy. 


\section{Materials and Methods}

This study involved 30 men aged 18-44 years. According to the WHO classification, all subjects belonged to the group of young people. The average age of the patients was $31.8 \pm 2.12$. All the patients selected for the study were divided into two groups. Group I included 14 patients with congenital central nervous system abnormalities, and group II included 16 patients with posttraumatic epilepsy. The median time since the injury occurred is 1.6 years. In addition, a control group of 15 practically healthy men of comparable age was formed. The men with significant somatic pathology, acute and chronic, as well as with brain tumors, acute traumatic brain injuries and acute infectious diseases of various etiologies did not include in this study. All patients underwent a neurological examination according to the generally accepted method, as well as a general examination and determination of basic physical data to exclude actual somatic pathology. The study included neuropsychological testing using the Quality of life in epilepsy questionnaire-31 (QOLIE-31), a validated questionnaire "International Index of Erectile Function" (IIEF). The surrogate criterion for male fertility was a spermogram. A biochemical blood test was also performed. EEG and EEG-video monitoring were used as screening methods.

We analyzed the quality of life (QOL) of patients with epilepsy, the nature of epileptic seizures, and also took into account other complaints, anamnestic data, a genealogical history to identify a genetic predisposition to epilepsy. The form of epilepsy and the type of epileptic seizures were determined according to the International Classification of Epilepsy and Epileptic Seizures (ILAE, 2017) [3]. The Student's T-test and Pearson's consensus test $(\chi 2)$ were used for statistical processing.

\section{Results}

The analysis of the data of 30 patients (100\%) on the QOLIE-31 scale showed that 3 people $(10 \%)$ rated their QOL as "very low". "Low" and "average" QOL scores were obtained from 9 (30\%) and $12(40 \%)$ patients, respectively. In $6(20 \%)$ people, the parameters were located in the intervals of "good" QOL (Figure 2). No ratings of "high" quality of life were obtained in comparison with the control group. The analysis of data from 15 apparently healthy young men in the control group on the QOLIE-31 scale showed that $1(6 \%)$ people rated their QOL as "average", $9(60 \%)$ people - as "good" and 5 (34\%) - as "high" QOL (Figure 2 \& 3).

All men were divided according to the form of epilepsy according to the International Classification of Epilepsy and Epileptic Seizures (Figure 4).

It was found in the course of the study that the average score on the ICEF questionnaire was 13.6 in group I, which corresponds to moderate erectile dysfunction. At the same time, there was a statistically significant difference with the control group $(p<0.01)$. This measure was 22.8 in group II, which actually indicates a normal erection. There was no significant difference with the control group in this indicator $(p>0.05)$. The $11(78.6 \%)$ patients from group I noted the absence of pronounced morning erections and weak masturbation erections, which allowed to exclude psychogenic erectile dysfunction. The men from the control group and group II noted the preservation of morning erections. The erectile dysfunction is a common finding in young men suffering from epilepsy. Also, young men from group I were significantly more likely to have asthenozoospermia (71.4\% compared to $20 \%$ of the control group; $\mathrm{p}<0.05)$. There was no statistically significant difference in group II with the control group.

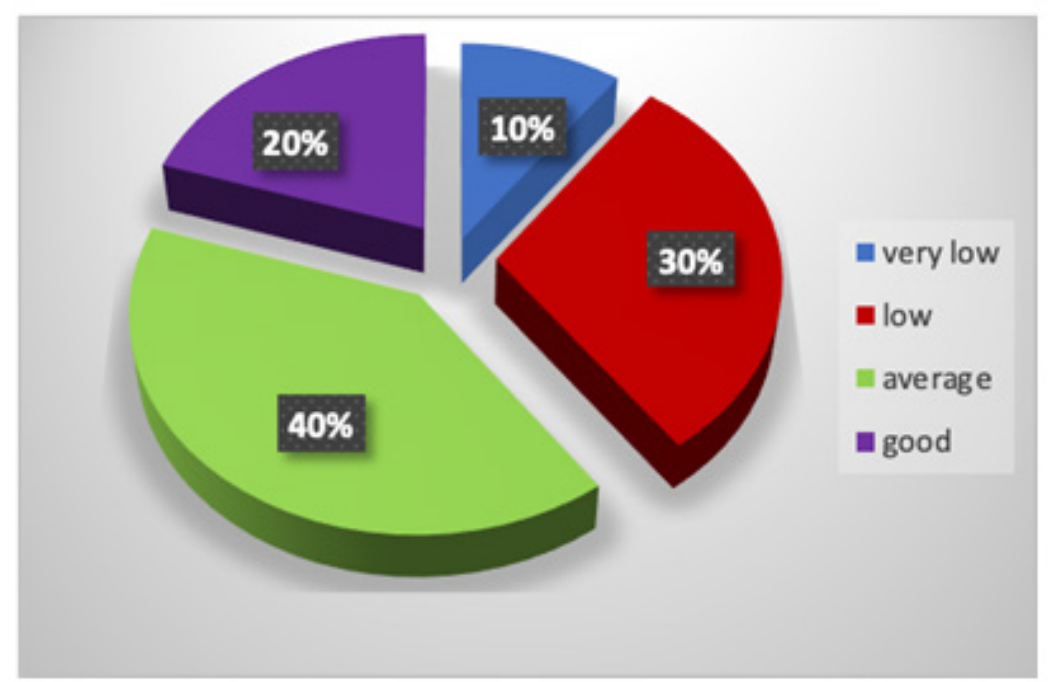

Figure 2: The quality of life for young men suffering epilepsy. 


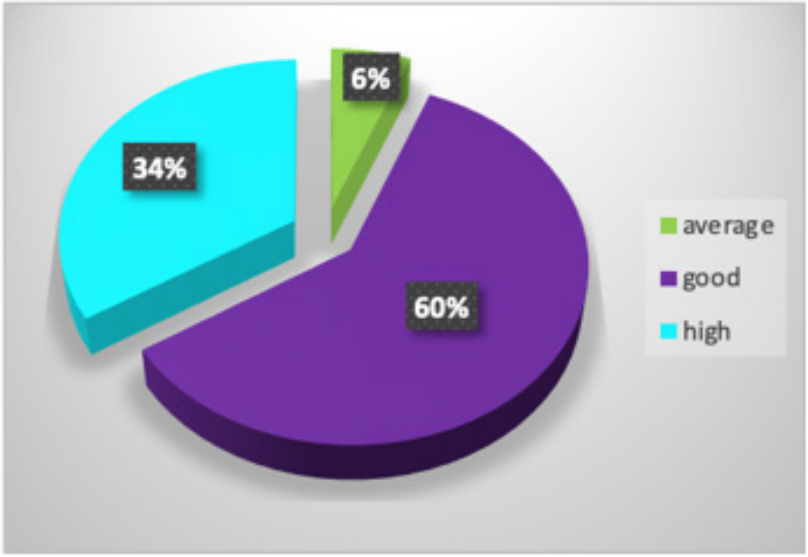

Figure 3: The quality of life for young men in the control group.

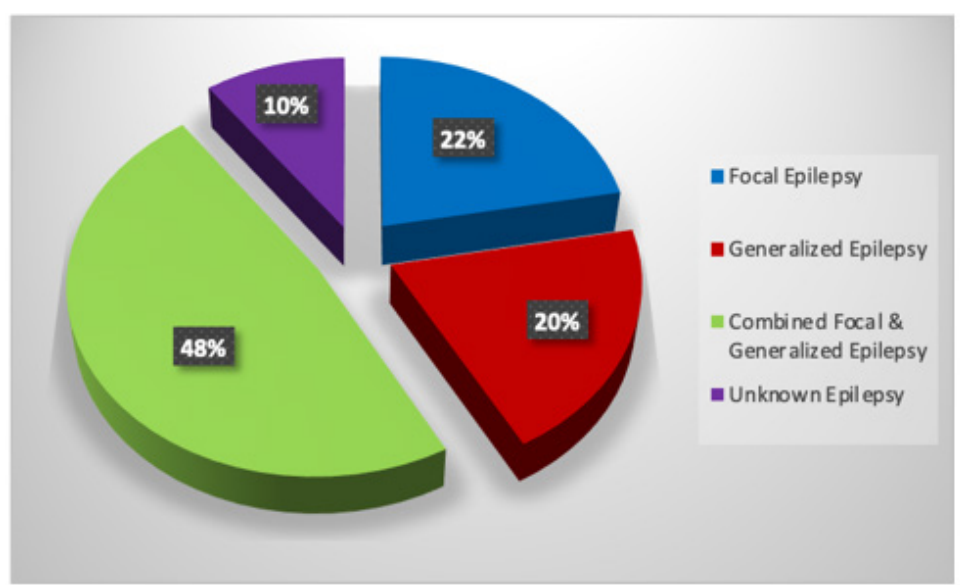

Figure 4: Number of patients depending on the form of epilepsy.

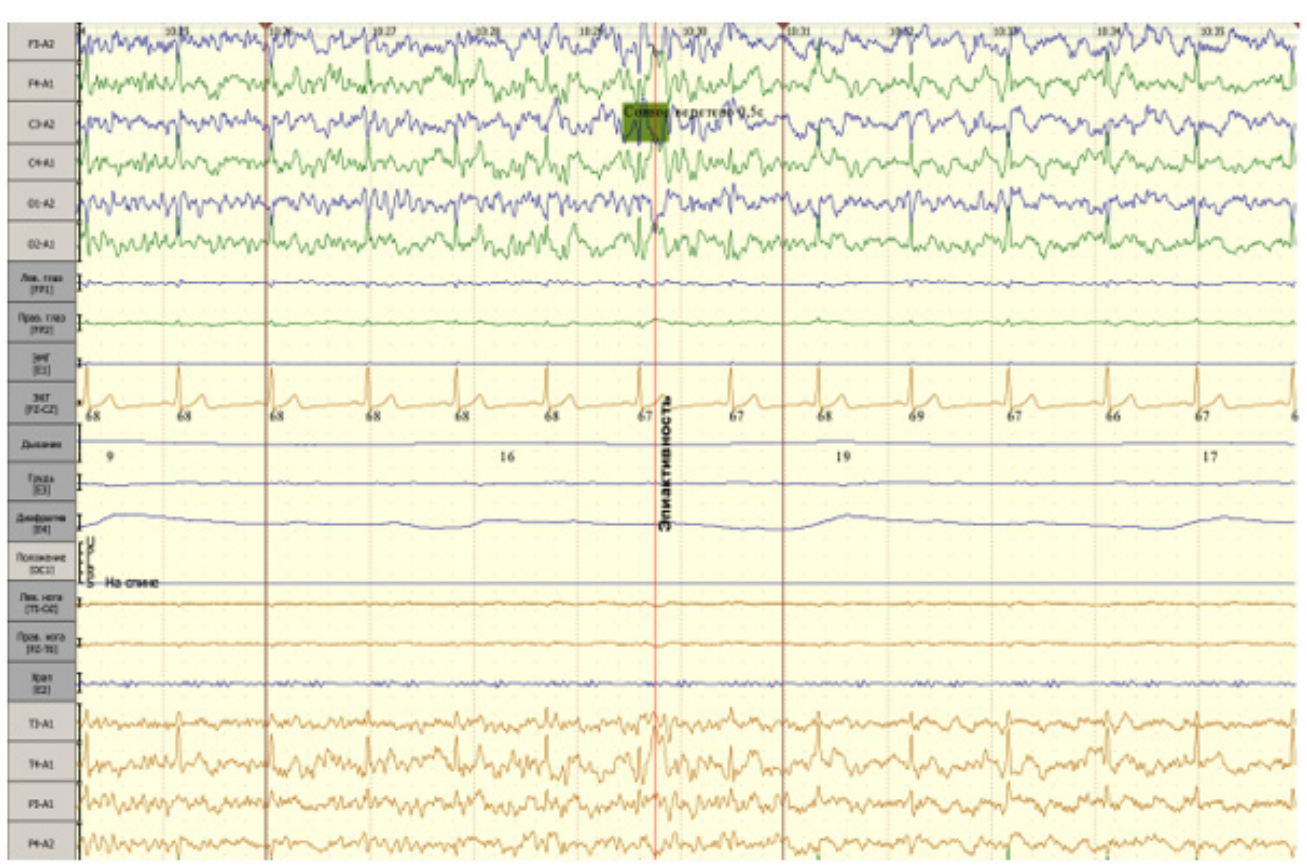

Figure 5: Patient T., male, 34 y.o. A fragment of the EEG. 
The EEG recorded both epileptiform activity with a focus in the left frontocentral and right temporal regions, and a decrease in cortical resistance to external stimuli, the presence of corticalsubcortical disharmonies in patients with epilepsy, which is an aggravating factor in the development of erectile dysfunction. The absence of a correlation between the severity of neurodynamic disorders and erectile dysfunction is a confirmation of the background, rather than the leading cause of cortical disorders in the development of erectile dysfunction in these patients (Figure 5).

However, it should not be forgotten that the reproductive system is affected not only by epilepsy, but also by the use of AEDs. This study compared the levels of testosterone, estradiol, luteinizing hormone, follicle-stimulating hormone, and gonadal efficiency (the ratio of testosterone/luteinizing hormone). All young men were divided into four groups: group I - patients who took carbamazepine, group II - men who took valproic acid drugs, group III - lamotrigine and group IV - control group. Patients treated with carbamazepine, valproic acid, and lamotrigine had significantly lower average testosterone levels than the control group $(\mathrm{p}<0.01)$. However, patients who took lamotrigine had significantly higher average testosterone levels than patients in the carbamazepine and valproate group $(p<0.01)$ and the control group $(p<0.05)$. No significant differences in the average estradiol level were found between the groups. The ratio of testosterone to luteinizing hormone in the control group was higher than in the other groups $(\mathrm{p}<0.01)$. On the other hand, this value was higher in the lamotrigine group than in the carbamazepine and valproate groups $(\mathrm{p}<0.01)$. In the carbamazepine and valproate groups, there was no significant difference in the ratio of testosterone/luteinizing hormone. Thus, as a result of this study, it was found that the average level of reproductive hormones was lower in the lamotrigine group compared to the control group, among traditional antiepileptic drugs, lamotrigine has the least negative effect on the level of reproductive hormones.

\section{Conclusions}

This study indicates the diversity of reproductive disorders associated with epilepsy. The comorbid conditions often progress and their impact on the health and quality of life of the patient becomes more significant in comparison with epilepsy. The quality of life of patients with epilepsy is significantly reduced. The perception of the quality of life of patients with epilepsy is more dependent on emotional well-being, cognitive and social functioning. According to the results of the study, it was found out that erectile dysfunction and impaired fertility are more common in people with epilepsy since childhood and adolescence. This may be due to profound changes in neuroendocrine regulation under the influence of a long-term epileptogenic focus in the brain and prolonged exposure to AEDs. In contrast, among patients with post-traumatic epilepsy and a short duration of the disease, erectile dysfunction is not more common than in the general population.

\section{Acknowledgement}

None.

\section{Conflicts of Interests}

Authors declare the absence of any conflict of interests and their own financial interest that might be construed to influence the results or interpretation of their manuscript.

\section{References}

1. Electronic source: http://www.who.int

2. Electronic source: http://www.moz.gov.ua

3. Electronic source: http://www.ilae.org

4. Franceschi M, Perego L, Cavagnini F, Cattaneo A, Invitti C, et al. (1984) Effects of long-term antiepileptic therapy on the hypothalamic-pituitary axis in men. Epilepsia 25(1): 46-52.

5. Geisler J, Engelsen B, Berntsen H, Geisler S, Lonning P (1997) Differential effect of carbamazepine and valproate monotherapy on plasma levels of oestrone sulphate and dehydroepiandrosterone sulphate in male epileptic patients. J Endocrinology 153(2): 307-312.

6. Herzog AG, Drislane FW, Schomer DL, Pennell PB, Bromfield EB, et al. (2004) Differential antiepileptic drug effects on sexual function and reproductive hormones: interim analysis of a comparison between lamotrigine and enzyme-inducing antiepileptic drugs. Epilepsia 45: 764-768.

7. Isojarvi J, Pakarinen A, Ylipalosaari P, VV Myllylä (1990) Serum hormones in male epileptic patients receiving anticonvulsant medication. Arch Neurol 47: 670-676.

8. Leach MJ, Randall AD, Stefani A, et al. (2002) Lamotrigine. Mechanisms of action. In: Antiepileptic Drugs. In: R Levy, R Mattson, B Meldrun, E Perucca $\left(5^{\text {th }}\right.$ edn). Philadelphia 363-369.

9. MacPhee G, Larkin J, Butler E, GH Beastall, MJ Brodie (1988) Circulating hormones and pituitary responsiveness in young epileptic men receiving long-term antiepileptic medication. Epilepsia 29: 468-475.

10. Mazdeh M, Heidari M, Taheri M, Ghafouri-Fard S (2020) Anticonvulsant drugs effects on sex hormone levels and sexual function in men with epilepsy. Future Neurology 15. 2.

11. Montouris G, Morris G (2005) Reproductive and sexual dysfunction in men with epilepsy. Epilepsy \& Behavior 7: 7-14.

12. Røste LS, Taubøll E, Mørkrid L, T Bjørnenak, E R Saetre, et al. (2005) Antiepileptic drugs alter reproductive endocrine hormones in men with epilepsy. Eur J Neurol 12(2): 118-124.

13. Wu D, Chen L, Ji F, Yang Si, Hongbin Sun (2018) The effects of oxcarbazepine, levetiracetam, and lamotrigine on semen quality, sexual function, and sex hormones in male adults with epilepsy. Epilepsia 59: $1344-1350$

14. Yerby M, McCoy G (1999) Male infertility: possible association with valproate exposure. Epilepsia 40(4): 520-521.

15. Zhao S, Wang X, Wang Y, Junjie Xu, Gang Zhu, et al. (2018) Effects of valproate on reproductive endocrine function in male patients with epilepsy: A systematic review and meta-analysis. Epilepsy Behavior 85: 120-128. 